\title{
Evaluación de un modelo de intervención domiciliaria en remoto con personas mayores y su entorno
}

\section{Karmele Acedo Gil}

Grupo Servicios Sociales Integrados

kacedo@grupossi.es

\section{Itziar Álvarez Lombardía}

Grupo Servicios Sociales Integrados

\section{Susana Moreno Barrio}

Grupo Servicios Sociales Integrados

\section{Ana Vega Carrillo \\ Grupo Servicios Sociales Integrados}

\section{Iraitz Manterola del Puerto}

Tecnalia Research and Innovation

\section{Javier Arcas Ruiz-Ruano}

Tecnalia Research and Innovation

\section{Gabriel Gaminde Pérez}

Tecnalia Research and Innovation

Servicios Sociales Integrados Kooperatiba Elkarteak eta Tecnalia Research \& Innovation erakundeak 20122013 urteen artean burutu zituzten hiru proiektu pilotu -Urrutiko Sostengu Psikosoziala, Urrutiko Estimulazio Kognitiboa eta Urrutiko Konpainia emate Soziala-, eta horien bidez xede zen balioztatzea Urrutiko Etxez Etxeko Eskuartze Eredu bat. Aipaturiko proiektu horiek zuzentzen ziren pertsona adindu, zaintzaile eta familia-zaintzaileentzat, eta etxez etxeko laguntza-zerbitzuko zuzeneko arretako langile eta konpainia emate sozialeko langileentzat; eta horren bidez, etxebizitzetatik partaide ziren pertsonak bideokonferentziaz harremanean jartzeko aukera izaten zuten, erabilerraza den ordenagailu bat erabiliz, eta modu horretan dagokion arreta eskainiz. Antzeman ziren arrakastaren giltzarritzat ulertutako faktore batzuek aipaturiko hiru proiektu pilotu horietan, eta horiek azaltzen dira artikulu honetan.

\section{GAKO-HITZAK:}

Pertsona adinduak, zaintzaileak, etxez etxeko arreta, sostengu psikosoziala, estimulazio kognitiboa, konpainia emate soziala, telelaguntza.
Durante 2012-2013, Grupo Servicios Sociales Integrados y Tecnalia Research \& Innovation llevaron a cabo tres proyectos piloto -Apoyo Psicosocial en Remoto, Estimulación Cognitiva en Remoto, y Acompañamiento Social en Remoto- con los que se perseguía validar un Modelo de Intervención Domiciliaria en Remoto. Dichos proyectos se dirigían a mayores, cuidadores y cuidadoras familiares, y personas trabajadoras de atención directa del servicio de ayuda a domicilio y del programa de acompañamiento social; y permitían a las personas participantes, desde sus domicilios, ponerse en contacto con profesionales mediante videoconferencia, a través de un ordenador de fácil manejo, y recibir la atención correspondiente. Se identificaron una serie de factores que se consideraron clave en el éxito de la implantación de los tres proyectos piloto citados, tal como se refleja en el presente artículo.

\section{Palabras clave:}

Personas mayores, cuidadoras, atención domiciliaria, apoyo psicosocial, estimulación cognitiva, acompañamiento social, teleasistencia. 


\section{Introducción}

ZureanTek es una alianza estratégica entre la cooperativa Grupo Servicios Sociales Integrados (en adelante, Grupo SSI) y el centro de investigación aplicada Tecnalia Research \& Innovation (en adelante, Tecnalia), cuya misión es innovar en la atención domiciliaria a través de servicios o programas con base tecnológica, dirigidos a personas en situación de envejecimiento, cronicidad, discapacidad o dependencia ${ }^{1}$, y sus familias. Durante 2012-2013, en el marco de esta alianza estratégica, se llevaron a cabo tres proyectos piloto, financiados por la Fundación para la Investigación Sociosanitaria Etorbizi, que perseguían validar un Modelo de Intervención Domiciliaria en Remoto (MIDER):

- Apoyo Psicosocial en Remoto (APRE).

- Estimulación Cognitiva en Remoto (ESCORE).

- Acompañamiento Social en Remoto (SAR).

El proyecto APRE se dirigía a tres colectivos: personas mayores, cuidadores y cuidadoras familiares, y personas trabajadoras de atención directa del servicio de ayuda a domicilio (SAD) o del programa de acompañamiento social. Los proyectos ESCORE y SAR, por su parte, se dirigían exclusivamente a personas mayores. De manera general, las personas que participaron en los tres proyectos contactaban desde sus domicilios, mediante videoconferencia, con profesionales especializados/as en psicología o acompañamiento social, a través de un ordenador de fácil manejo, y recibían la atención correspondiente.

El fin último de estos proyectos piloto fue mejorar la calidad de vida de las personas mayores, favoreciendo su permanencia en el entorno personal, familiar y social, así como, la de sus familias. En cuanto a los objetivos generales:

- Los del proyecto APRE eran diferentes para cada uno de los colectivos a los que iba dirigido:

- Que las personas mayores mejorasen o mantuvieran las funciones psicoafectivas.

- Que las personas cuidadoras familiares previnieran o desarrollaran estrategias de manejo de las consecuencias negativas derivadas del cuidado familiar.

- Que las auxiliares de ayuda a domicilio y acompañantes previnieran o desarrollaran estrategias de prevención o manejo del síndrome de burnout.

- El proyecto ESCORE buscaba prevenir o retrasar el deterioro de las funciones cognitivas de las personas mayores.

- El proyecto SAR se proponía que las personas mayores dispusieran de apoyo social.

${ }^{1}$ En este artículo se empleará el término ‘personas mayores' para hacer referencia a personas en situación de envejecimiento, cronicidad, discapacidad o dependencia.
El pilotaje de las tres experiencias se llevó a cabo en coordinación con los municipios del territorio histórico de Bizkaia en los que el Grupo SSI gestionaba el SAD, el programa de acompañamiento social o el programa foral Zainduz (Abanto-Zierbena, Amorebieta-Etxano, Arrigorriaga, Bilbao, Muskiz y Ugao-Miraballes).

\section{Contextualización}

Varios factores sociodemográficos caracterizan el contexto en el que se enmarcaban las experiencias piloto. Uno de ellos es el envejecimiento progresivo de la población, es decir, el incremento de la proporción de personas mayores respecto a la población total. Si en 1999 la población mayor de 64 años de la Comunidad Autónoma del País Vasco (CAPV) suponía el 16,8\% del total, en 2013 era ya el 20,3\%. Además, entre las personas mayores se da una mayor proporción de mujeres $(57,9 \%)$ que de hombres $(42,1 \%)$ -lo que se denomina feminización del envejecimiento-, así como un crecimiento de la población mayor de 79 años, colectivo que representa el 31,5\% de los y las mayores (INE, 2013). Las proyecciones de población revelan que la tendencia de aumento de este colectivo se mantendrá en los próximos años: se prevé que, para 2050, las personas mayores de 64 años representen el 38,8\% del total de la población de la CAPV (Eustat, 2012).

En cuanto a las situaciones de cronicidad y dependencia, es entre las personas mayores, y concretamente las mujeres, donde se da una mayor prevalencia. Los datos reflejan que el $85 \%$ de las personas mayores de 64 años padecen alguna enfermedad crónica (Gobierno Vasco, 2010), y que entre todas las personas dependientes de la CAPV, el 76,2 \% tienen más de 64 años (Gobierno Vasco, 2009).

En general, cuando los y las mayores necesitan ayuda, prefieren permanecer en su domicilio el mayor tiempo posible y contar con la implicación tanto de la familia como de profesionales (Gobierno Vasco, 2011a). La familia es la principal proveedora de cuidados a las personas mayores (Imserso, 2005). El cuidado suele recaer en una única persona del sistema familiar, el cuidador o la cuidadora principal, que es quien asume la responsabilidad de aquél. Esta función ha venido correspondiendo, casi en exclusiva, a las mujeres de la familia, que suponen el $77,3 \%$ del total de las personas cuidadoras de la CAPV (Gobierno Vasco, 2007). La situación de cuidado suele ser continuada y prolongada en el tiempo, y representa, para quien lo ejerce, una serie de costes personales que afectan a las diferentes esferas de su vida (Crespo y López, 2007).

El SAD es un recurso social relevante en el cuidado de las personas mayores $y$, de hecho, es el servicio más utilizado por las personas mayores de 59 años, por delante de la teleasistencia o los centros de día (Gobierno Vasco, 2011b). Las personas que trabajan en el SAD, principalmente mujeres, pueden llegar 
a presentar el denominado síndrome de burnout, una alteración psicológica característica de las y los profesionales que mantienen una relación directa, constante e intensa con las personas beneficiarias de su trabajo (Conde Morala, 2006).

Los proyectos piloto que nos ocupan nacieron para dar respuesta a una serie de necesidades específicas de los tres colectivos destinatarios, detectadas en los diferentes servicios sociales que gestiona el Grupo SSI. En relación al colectivo de personas mayores, se observó la importancia de una atención integral que abordara las diferentes dimensiones de la persona desde un enfoque biopsicosocial. En el SAD se realizan tareas de atención personal y domésticas, pero parecía necesario llevar a cabo intervenciones específicas en las áreas cognitiva, psicoafectiva, relacional y de ocio. En cuanto al colectivo de cuidadores y cuidadoras familiares, era importante seguir ofreciendo apoyo en lo concerniente a estrategias de cuidado de la persona mayor, y especialmente, de autocuidado de la propia persona cuidadora. Y respecto al colectivo de auxiliares de ayuda a domicilio y acompañantes, se vio la necesidad de actuar, tanto de manera preventiva como de apoyo, ante el estrés laboral que se puede derivar del trabajo continuado con personas. De manera general para los tres grupos, se constató la necesidad de diversificar los servicios que se prestaban, ofrecer intervenciones individualizadas y personalizadas, con una mayor flexibilidad, y además realizarlas en el marco del domicilio.

Para dar respuesta a esas necesidades, se optó por ofrecer servicios de apoyo psicosocial, estimulación cognitiva y acompañamiento social de manera remota. Dichos servicios ya se venían prestando en el domicilio de manera presencial, pero, por su coste, sólo se podía llegar a un número reducido de personas. Por ello, se decidió ensayar una nueva forma de prestarlos, partiendo de la hipótesis de que la tecnología podía ser un medio adecuado, de menor coste y mayor alcance en cuanto a número de personas beneficiarias.

\section{Implementación}

La implementación de los tres proyectos piloto se llevó a cabo en tres fases.

\subsection{Fase 1: planificación y preparación}

- Especificación de los requisitos de los equipos y de la aplicación web: el objetivo de esta tarea fue analizar qué requisitos, tanto funcionales y técnicos como de usabilidad, debían cumplir los ordenadores y la aplicación web para responder al perfil y necesidades de las personas participantes.

- Desarrollo de la plataforma tecnológica: se optó por un desarrollo iterativo e incremental de una aplicación web basada en videoconferencia, comenzando por una implementación simple de los requisitos, para después ir introduciendo mejoras en el diseño.

- Compra de equipos para participantes y contratación de la conexión a Internet: se compraron los ordenadores y se contrató a una empresa proveedora la conexión a Internet que se instalaría en los domicilios de las personas participantes que no disponían de ella (el 90\%).

- Formación del equipo coordinador y los/as profesionales de intervención: se formó sobre el uso del ordenador y de la aplicación web al equipo coordinador de los proyectos piloto, así como a las y los profesionales que llevarían a cabo la intervención directa.

- Validación de la plataforma tecnológica: el objetivo de esta tarea fue evaluar la aplicación web antes de llevarla a los domicilios de las personas participantes. Este proceso se realizó en dos fases. En la primera, se simuló una intervención en remoto con tres personas, en un entorno controlado (las instalaciones del Grupo SSI). En la segunda prueba, se implementó el proyecto APRE con un número reducido de auxiliares de ayuda a domicilio y acompañantes, en un entorno controlado y durante tres semanas. En ambos casos, se obtuvo retroalimentación y se llevaron a cabo las modificaciones técnicas pertinentes, lo que permitió elaborar una versión estable de la plataforma tecnológica, que se utilizó a lo largo de las experiencias.

- Diseño de los criterios de inclusión de las personas participantes: se establecieron los criterios de inclusión que debían cumplir las y los participantes, que en todos los casos serían usuarias o trabajadoras de los servicios sociales que gestionaba el Grupo SSI (Cuadro 1). Se consideró también tomar en cuenta una serie de condiciones de éxito que garantizaran el adecuado desarrollo de los proyectos piloto. Éstas estaban relacionadas con las características de las personas participantes (Cuadro 2) y, debido a que el despliegue de los proyectos piloto se iba a realizar en los domicilios, también con las condiciones de la vivienda (Cuadro 3).

- Diseño del modelo de intervención: el modelo de intervención se diseñó de tal manera que, en el proyecto APRE, las personas recibirían ocho sesiones de atención psicosocial, de una hora de duración, una vez a la semana; en el proyecto ESCORE, quince sesiones de estimulación cognitiva, de una hora de duración, tres veces por semana; y en el proyecto SAR, ocho sesiones de acompañamiento social, de una hora de duración, dos veces a la semana.

- Diseño del sistema de evaluación. Se diseñó un sistema que permitiera evaluar los proyectos piloto mediante un proceso de recogida, volcado y análisis de la información en torno a cuatro variables: eficiencia, eficacia, adecuación y utilidad. En esta tarea, se llevó a cabo el diseño y elaboración de los indicadores y las herramientas de evalua- 


\begin{tabular}{|c|c|c|c|}
\hline Colectivo & APRE & ESCORE & SAR \\
\hline Personas mayores & $\begin{array}{l}\text { - Ausencia de deterioro cognitivo } \\
\text { o de carácter leve. } \\
\text { - Capacidad para utilizar de } \\
\text { forma autónoma el dispositivo o } \\
\text { requerir para ello apoyo leve. } \\
\text { - Dificultad para acceder al exte- } \\
\text { rior del domicilio. } \\
\text { - Necesidad de intervención } \\
\text { psicológica de intensidad baja } \\
\text { o media. }\end{array}$ & $\begin{array}{l}\text { - Ausencia de actividades de } \\
\text { estimulación cognitiva. } \\
\text { - Ausencia de trastorno depresivo } \\
\text { o de carácter leve. } \\
\text { - Capacidad para leer y escribir. } \\
\text { - Capacidad para utilizar de } \\
\text { forma autónoma el dispositivo o } \\
\text { requerir apoyo leve. } \\
\text { - Puntuación } \geq 14 \text { en la prueba } \\
\text { Mini Mental State Examination } \\
\text { de Folstein. }\end{array}$ & $\begin{array}{l}\text { - Ausencia de trastorno } \\
\text { depresivo o de carácter leve. } \\
\text { - Capacidad para utilizar } \\
\text { de forma autónoma el } \\
\text { dispositivo o requerir para } \\
\text { ello apoyo leve. } \\
\text { - Escasa red de apoyo social. }\end{array}$ \\
\hline Personas cuidadoras familiares & $\begin{array}{l}\text { - Capacidad para utilizar de forma } \\
\text { autónoma el dispositivo. } \\
\text { - Dificultad para acudir a activida- } \\
\text { des de apoyo grupal o requerir } \\
\text { apoyo individual. }\end{array}$ & - & - \\
\hline Personas trabajadoras & $\begin{array}{l}\text { - Capacidad para utilizar de forma } \\
\text { autónoma el dispositivo. } \\
\text { - Haber manifestado síntomas } \\
\text { relacionados con el síndrome } \\
\text { del burnout o haber sido } \\
\text { identificada por las coordina- } \\
\text { doras del equipo de auxiliares y } \\
\text { acompañantes. }\end{array}$ & - & - \\
\hline
\end{tabular}

Fuente: Elaboración propia.

Cuadro 2. Características de las personas participantes

- Ausencia de problemas auditivos o de carácter leve.

- Ausencia de problemas visuales o de carácter leve.

- Ausencia de trastornos mentales.

- Autonomía para desplazarse en el interior del domicilio.

- Carácter comprometido.

- Familia implicada y con capacidad de apoyar a la persona.

- Motivación y actitud positiva y abierta hacia el uso de tecnologías.

- Movilidad de miembros superiores.

Fuente: Elaboración propia.

ción para la recogida de información, y el diseño y elaboración de una base de datos en formato Excel para el volcado de aquélla.

- Diseño del protocolo de actuación ante incidencias: se definió un protocolo de actuación ante incidencias, que se socializó entre todas las personas implicadas.

- Diseño y elaboración de los materiales: se llevó a cabo el diseño y elaboración de los materiales informativos y de comunicación sobre los proyectos piloto; de los manuales formativos sobre el manejo del ordenador y la plataforma tecnológica; y de los materiales de intervención que se emplearían en los proyectos ESCORE y SAR.

- Tramitación de aspectos éticos: se elaboró y presentó la documentación necesaria al Comité Ético de Investigación Clínica de Euskadi, cuyo dictamen fue favorable a la ejecución de las experiencias piloto.

- Coordinación con agentes intervinientes: se realizaron reuniones con las personas coordinadoras del SAD, del programa de acompañamiento social y del programa foral Zainduz, con las auxiliares de ayuda a domicilio, con el personal

\section{Cuadro 3. Condiciones de la vivienda}

- Cobertura de ADSL.

- Instalación eléctrica adecuada.

- Mesa y silla para la colocación del dispositivo en un espacio tranquilo.

- Teléfono fijo (preferentemente inalámbrico).

- Toma de corriente cerca de la mesa.

Fuente: Elaboración propia.

de recursos humanos del Grupo SSI y con el personal responsable y técnico de los departamentos de Acción Social de los municipios en las que se desplegaron los proyectos piloto. En estas reuniones, se informó sobre dichos proyectos y se implicó a esas personas en la selección de las y los participantes.

\subsection{Fase 2: despliegue de los proyectos piloto}

- Selección de la muestra: en primer lugar, diferentes agentes (Cuadro 4) elaboraron una primera ficha con posibles personas candidatas, de acuerdo con los criterios de inclusión y las condiciones de éxito referidas anteriormente. En segundo lugar, la empresa proveedora de conexión a Internet comprobó que los domicilios de la ficha disponían de cobertura ADSL (no se aportaron datos identificativos de las personas) para poder participar en la experiencia, lo que permitió obtener una segunda ficha con posibles personas candidatas. En tercer lugar, se realizó un primer contacto telefónico con estas personas, en el que se daba una breve explicación del proyecto piloto, 
con el objetivo de que pudieran manifestar su interés inicial por participar en él. Finalmente, la persona responsable de cada proyecto piloto contactó nuevamente con esas personas a fin de concertar una cita. En esta visita se explicó detalladamente el proyecto, se hizo entrega de documentación informativa, se comprobó el cumplimiento de las condiciones de la vivienda y se confirmó el interés inicial de la persona por participar en el proyecto, para después formalizar la adhesión mediante la firma del consentimiento informado.

- Evaluación inicial de la muestra: se recogió información sobre las personas participantes (datos sociodemográficos, entorno sociofamiliar, salud física y psicológica, y expectativas sobre el proyecto) y se aplicaron pruebas de evaluación estandarizadas (Cuadro 5).

- Diseño del plan de intervención personalizado: se elaboraron los planes personalizados, que recogían los objetivos de la intervención y las actividades para alcanzarlos.

- Altas de la conexión a Internet e instalación del ordenador en los domicilios: se dio de alta la conexión a Internet tras las instalaciones necesarias y se instaló el ordenador en el domicilio de las personas participantes.

- Apoyo pedagógico a las personas participantes: la persona responsable de cada proyecto impartió una formación específica sobre el uso del dispositivo y de la aplicación tecnológica, con una prueba de videoconferencia de forma presencial en el domicilio de las y los participantes, y la entrega de un manual adaptado a las características de cada colectivo. En ese momento se entregó también el calendario de intervención, con las fechas previstas y los teléfonos de contacto ante incidencias.

- Apoyo sociotecnológico a las personas participantes: se proporcionó mantenimiento y soporte técnico durante la ejecución de los proyectos piloto. En la primera sesión de intervención, se visitaron los domicilios con el objetivo de aportar seguridad a las personas participantes. Durante el transcurso de todas las sesiones, el equipo del Grupo SSI y el de Tecnalia realizaron conjuntamente guardias en remoto, que permitieron proveer de asistencia técnica y actuaciones en tiempo real ante incidencias.

- Seguimiento: se llevó a cabo el seguimiento de las sesiones, por un lado, a través de la información que recogían las y los profesionales de la intervención en la plataforma tecnológica, y por otro, mediante contactos telefónicos periódicos por parte del equipo coordinador.

- Evaluación final de la muestra: para la evaluación final de la muestra, se recogieron las mismas variables que al inicio, así como el grado de satisfacción sobre distintas cuestiones relacionadas con las sesiones, la persona profesional, el ordenador y la gestión del proyecto piloto.

- Retirada de los equipos en los domicilios y tramitación de bajas en la conexión a Internet: el último paso de la implementación de los proyectos piloto fue la retirada de los equipos en los domicilios y la tramitación de bajas en la conexión a Internet.

\begin{tabular}{|l|l|}
\hline \multicolumn{2}{|l|}{ Cuadro 4. Participantes en la selección de la muestra, por proyecto } \\
\hline Selección de & Realizada por \\
\hline Personas mayores & $\begin{array}{l}\text { • Auxiliares de ayuda a domicilio, con validación de las coordinadoras del SAD. } \\
\text { - Apoyo de las responsables de los departamentos de Acción Social. }\end{array}$ \\
\hline Personas cuidadoras familiares & $\begin{array}{l}\text { - Coordinadoras del programa foral Zainduz. } \\
\text { - Apoyo de las responsables de los departamentos de Acción Social. }\end{array}$ \\
\hline Auxiliares/acompañantes & $\begin{array}{l}\text { - Coordinadoras del SAD y del programa de acompañamiento social. } \\
\text { - Recursos humanos del Grupo SSI. }\end{array}$ \\
\hline
\end{tabular}

Fuente: Elaboración propia.

\begin{tabular}{|c|c|c|c|}
\hline Colectivo & APRE & ESCORE & SAR \\
\hline Personas mayores & $\begin{array}{l}\text { - Cuestionario sobre exceso de } \\
\text { incapacidad. } \\
\text { - Escala de Ansiedad y Depresión } \\
\text { de Goldberg (EADG). } \\
\text { - Escala de Lawton y Brody. } \\
\text { - Índice de Barthel. } \\
\text { - Short Portable Mental State } \\
\text { Questionnaire de Pfeiffer } \\
\text { (SPMSQ). }\end{array}$ & $\begin{array}{l}\text { - Escala de Ansiedad y Depresión } \\
\text { de Goldberg (EADG). } \\
\text { - Mini Mental State Examination } \\
\text { de Folstein (MMSE). }\end{array}$ & $\begin{array}{l}\text { - Escala de Ansiedad y Depresión } \\
\text { de Goldberg (EADG). } \\
\text { - Escala ESTE de Soledad Social. } \\
\text { - Short Portable Mental State } \\
\text { Questionnaire de Pfeiffer } \\
\text { (SPMSQ). }\end{array}$ \\
\hline Personas cuidadoras familiares & $\begin{array}{l}\text { - Escala de Ansiedad y Depresión } \\
\text { de Goldberg (EADG). } \\
\text { - Escala de Sobrecarga de Zarit. }\end{array}$ & - & - \\
\hline Personas trabajadoras & $\begin{array}{l}\text { - Escala de Ansiedad y Depresión } \\
\text { de Goldberg (EADG). } \\
\text { - Maslach Burnout Inventory } \\
\text { (MBI). }\end{array}$ & - & - \\
\hline
\end{tabular}

Fuente: Elaboración propia. 


\subsection{Fase 3: evaluación}

Los proyectos piloto fueron evaluados por todas las personas implicadas en su desarrollo, a través de entrevistas individuales y de grupos de discusión.

\section{Descripción de los equipos y la plataforma tecnológica}

\subsection{El hardware: los equipos}

Se facilitaron equipos con conexión a Internet tanto a los y las participantes como a las personas profesionales que realizaron la intervención. En el caso de las personas participantes, el ordenador seleccionado fue un MSI Wind Top AE2050, un equipo todo en uno, con pantalla táctil de 20 pulgadas, con cámara web, micrófono y altavoces integrados. En el caso de las personas participantes que tenían problemas de audición, se utilizaron auriculares con micrófono Logitech h110. De cara a facilitar el uso del equipo, se evitó el uso del teclado y el ratón, y se optó por la pantalla táctil como forma de interacción con el equipo.

Figura 1. Equipo informático utilizado por las personas participantes en los proyectos piloto

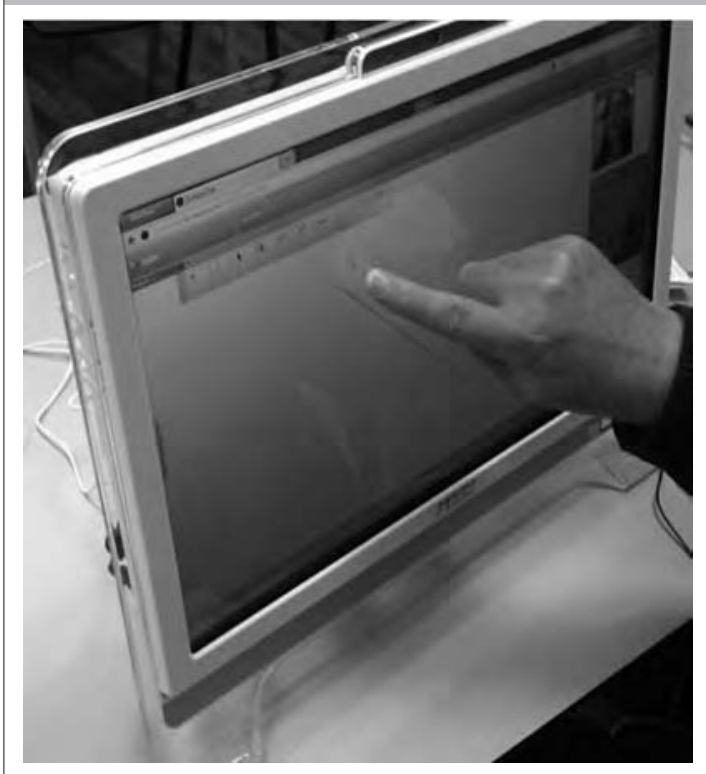

Fuente: Elaboración propia.

En cuanto a los equipos utilizados por las personas profesionales, debido a que este colectivo tenía conocimientos informáticos, se optó por la tradicional torre conectada a un monitor por cables.

Por lo que se refiere a la conexión a Internet en los domicilios de las personas participantes, se dieron de alta líneas de ADSL debido principalmente a una mayor garantía de ancho de banda y estabilidad en la conexión frente a la modalidad de Internet móvil.

\subsection{El software: la aplicación web}

La plataforma tecnológica utilizada se basó en una aplicación web desarrollada por Tecnalia, a través de la cual se llevaban a cabo las sesiones de intervención mediante videoconferencia entre las personas participantes y los y las profesionales. La aplicación disponía de las siguientes funcionalidades:

- Videoconferencia individual y múltiple: la plataforma, desarrollada a partir del código abierto de la aplicación OpenMeetings, permitía la comunicación remota visual y verbal entre la persona participante y el o la profesional mediante videoconferencia. Asimismo, incorporaba una herramienta para realizar videoconferencias múltiples. El número máximo de personas que se podían conectar al mismo tiempo era de 200.

- Pizarra virtual: la videoconferencia se acompañaba de una pizarra virtual que compartían tanto la persona participante como la o el profesional. La interacción se realizaba por medio de una barra de herramientas que permitía escribir, dibujar o señalar.

- Importación de documentos: la importación de documentos (para las fichas de estimulación cognitiva, por ejemplo) era instantánea, gracias a un explorador de archivos integrado. El sistema permitía importar archivos de distinto tipo (pdf, doc, odt, ppt, png o jpg, entre otros), que quedaban almacenados en la plataforma para una posterior reutilización. A su vez, cada pizarra podía ser tratada como un archivo, ya que todo su contenido se podía guardar también en la propia plataforma en el caso de que se decidiera volver a utilizar en una futura sesión de videoconferencia.

- Gestor de personas: la plataforma disponía de una herramienta para la gestión de las personas participantes por parte de los y las profesionales. Esta herramienta permitía elaborar, almacenar y recuperar los objetivos, metodología y actividades, así como la evaluación de cada sesión por parte de la persona profesional.

- Navegación: la navegación era sencilla e intuitiva, y constaba únicamente de tres pantallas para las personas participantes y de cuatro para las personas profesionales.

Asimismo, se llevaron a cabo una serie de adaptaciones para que el uso del ordenador fuera lo más sencillo posible:

- Arranque: se automatizó la apertura del navegador web, de forma que se accedía directamente a la pantalla de inicio de la aplicación web y las personas participantes no tenían que realizar ninguna acción para entrar en ella.

- Identificación de las personas: se diseñó un sistema de almacenamiento de claves a través del cual las personas sólo debían identificarse una única vez, introduciendo nombre de usuario y contraseña. Durante la instalación de los ordenadores 
en los domicilios, se procedió a entrar por primera vez en la página principal de la aplicación web y se introdujo el par usuario/contraseña correspondiente a las personas participantes, de modo que quedaba almacenada en los ordenadores.

- Escritorio. Se eliminaron todos los iconos del escritorio, dejándose únicamente dos iconos creados ad hoc: uno para el acceso directo a la aplicación web (por si se cerrara el navegador y hubiera que volver a acceder al sitio web), y otro para el apagado del ordenador (un solo toque en dicho icono ejecutaba la secuencia habitual de apagado ‘Menú inicio' + ‘Apagar' del sistema operativo Windows).

\section{Resultados}

En este apartado se describen los principales resultados de los proyectos piloto.

\subsection{Número y perfil de las personas participantes}

El número total de personas que participaron en los proyectos piloto hasta su término fue de 83 (78 mujeres y 5 hombres) [Tabla 1]. En el proyecto APRE, participaron 38 personas, todas mujeres, de las cuales 10 fueron mayores, 10 cuidadoras, y 18 auxiliares de ayuda a domicilio o acompañantes. En el proyecto ESCORE participaron 9 personas mayores, también todas ellas mujeres. En el proyecto piloto SAR participaron 36 personas ( 31 mujeres y 5 hombres).

En cuanto al perfil de las personas participantes, en el caso de las personas mayores, era el de una mujer, de entre 70 y 79 años, viuda, que vivía sola y contaba con estudios primarios. El perfil de las personas cuidadoras familiares era también el de una mujer, de entre 55 y 64 años, casada, que vivía en pareja o con otros/as familiares, que contaba con estudios primarios o secundarios, y que era hija de la persona dependiente, a la que llevaba cuidando entre 3 y 4 años. El perfil de las auxiliares y acompañantes era el de una mujer, de entre 45 y 49 años, casada, que vivía con otros/as familiares, que contaba con estudios primarios y que llevaba trabajando entre 0 y 5 años. Ninguno de los tres colectivos estaba familiarizado con el uso del ordenador. En cuanto a las bajas, se dieron 12 antes de iniciar la intervención en remoto, todas ellas mujeres, y 9 durante la intervención (8 mujeres y 1 hombre).

\subsection{Número de sesiones y horas de intervención}

El número total de horas de intervención en los tres proyectos piloto fue de 670 (Tabla 2). En el proyecto APRE, se llevaron a cabo 281 horas de intervención, 77 en el caso de las personas mayores, 77 con las cuidadoras, y 127 con las auxiliares y acompañantes. En el proyecto ESCORE, se realizaron 131 horas de intervención; y en el proyecto SAR, 258.

\subsection{Grado de consecución de objetivos}

El grado medio de consecución de los objetivos en el APRE fue del $90 \%$ en el caso de las personas mayores, del $92 \%$ en las cuidadoras, y del $78 \%$ en las auxiliares y acompañantes. En el ESCORE fue del $91 \%$, y en el SAR, del $92 \%$.

\subsection{Número de guardias e incidencias}

Durante la ejecución de los proyectos piloto, se realizaron un total de 670 horas de guardia. El número total de incidencias recogidas fue de 430 (Tabla 1).

Tabla 1. Incidencias, por proyecto y tipología (\%)

\begin{tabular}{|l|c|c|c|c|}
\hline Proyecto & Técnica & Manejo & Otras & Total \\
\hline APRE & 60 & 5 & 35 & 100 \\
\hline ESCORE & 84 & 5 & 10 & 100 \\
\hline SAR & 53 & 25 & 22 & 100 \\
\hline Total & 100 & 100 & 100 & 100 \\
\hline
\end{tabular}

Fuente: Elaboración propia.

\subsection{Grado de satisfacción}

El grado de satisfacción expresado por las personas participantes de los tres proyectos piloto fue elevado (Tabla 2). En particular, destaca la valoración del personal.

\subsection{Usabilidad}

Las personas participantes calificaron el equipo y la plataforma tecnológica como fáciles de usar, superando las expectativas que se tenían al respecto.

Tabla 2. Grado de satisfacción de las personas participantes, por proyecto y tipología de participantes (escala del 1 al 5)

\begin{tabular}{|l|c|c|c|c|c|}
\hline \multirow{2}{*}{ İtems } & \multicolumn{3}{|c|}{ APRE } & \multicolumn{2}{c|}{ ESCORE } \\
\cline { 2 - 6 } & Personas mayores & Personas cuidadoras & \multicolumn{2}{c|}{ Personas trabajadoras } & \multicolumn{2}{c|}{ Personas mayores } \\
\hline Servicio & 4,1 & 4,7 & 4,1 & 4,5 & 4,08 \\
\hline Profesional & 5,0 & 5,0 & 4,8 & 4,9 & 4,70 \\
\hline Equipo coordinador & 4,5 & 5,0 & 4,9 & 4,9 & 4,95 \\
\hline Organización & 4,4 & 4,7 & 4,2 & 4,27 \\
\hline Tecnología & 4,8 & 5,0 & 4,5 & 4,6 & 4,49 \\
\hline
\end{tabular}

Fuente: Elaboración propia. 


\section{Discusión}

\subsection{Conclusiones de las experiencias piloto}

Como se ha mencionado, las principales conclusiones de los proyectos piloto se extrajeron con base en cuatro variables: eficacia, eficiencia, adecuación y utilidad. En relación a la eficacia, la modalidad de intervención en remoto no parece influir negativamente en la consecución de los objetivos marcados, comparada con el formato presencial, si bien, parece importante tener en cuenta que en estas experiencias, al tratarse de proyectos piloto, el número de sesiones se consideró reducido para poder obtener cambios significativos en las personas participantes.

Por lo que se refiere a la adecuación y utilidad, se concluyó que las intervenciones desarrolladas en remoto parecen adecuadas para personas con capacidades físicas, cognitivas, psicoafectivas y relacionales preservadas. En el caso de las personas mayores, se observa que la modalidad de intervención remota puede ser eficaz para actuar en las áreas cognitiva, psicoafectiva, relacional y de ocio, pero parece poco útil para trabajar el mantenimiento o mejora de la autonomía personal. Respecto a las auxiliares y acompañantes, la modalidad en remoto puede ser muy útil y adecuada para trabajar la supervisión de casos. En cuanto al acompañamiento social, el tipo de actividades que se pueden realizar de manera remota en las sesiones parece limitado con respecto a la intervención presencial.

En lo referente a la eficiencia, se han identificado una serie de costes específicos derivados del desarrollo de cualquier intervención en remoto: inversión en equipos y programas, contratación e instalación de conexión a Internet, desplazamientos a domicilios para instalación y retirada de equipos, y mantenimiento y soporte técnico. Por otro lado, no se producen los costes de los desplazamientos de las y los profesionales de intervención a los domicilios de las personas participantes para llevar a cabo las sesiones en modalidad presencial.

En cuanto a las incidencias, el volumen no ha sido un obstáculo para el desarrollo de las intervenciones y no ha influido en el grado de satisfacción de las personas participantes y profesionales, lo que puede deberse principalmente a dos aspectos: por un lado, a que se informó previamente a las personas de que se trataba de un proyecto en fase experimental, por lo que podrían darse incidencias; y por otro, al apoyo sociotecnológico que se desplegó.

Respecto a las incidencias de manejo, fueron poco significativas y se considera que ello puede obedecer principalmente a la identificación adecuada de los requisitos del ordenador y de la plataforma tecnológica, y al apoyo pedagógico que se dio a las personas participantes para su uso. Las incidencias englobadas en la categoría 'Otras' estuvieron relacionadas fundamentalmente con la conexión a Internet, y en concreto con un ancho de banda insuficiente, en cuanto a velocidad de subida, para garantizar una videoconferencia de calidad. Éste se considera un factor externo al desarrollo de cualquier intervención en remoto, que depende del mercado y de los operadores que comercializan la conexión a Internet.

\subsection{Aspectos clave en la implantación de las experiencias piloto}

Se han identificado una serie de factores que se consideran clave en el éxito de la implantación de los tres proyectos piloto. Dichos factores están relacionados principalmente con el apoyo humano que se aportó a las personas que participaron en estas experiencias tecnológicamente mediadas, y que garantizaron su adhesión, implicación y permanencia en los proyectos piloto:

- La motivación: la implicación del equipo del SAD (coordinación y auxiliares) en el proceso de selección de las personas participantes inicialmente, $y$ durante el proceso de intervención en los domicilios posteriormente; en ambos casos, ejercieron un papel motivador en las personas mayores.

- El vínculo: generar un vínculo de confianza con las y los participantes, tanto por parte del equipo coordinador, como del o la profesional de intervención posibilita que la relación se perciba como cercana.

- El apoyo pedagógico: formar a las personas participantes en el uso del ordenador y de la aplicación web favorece que el número de incidencias de manejo sea poco significativo.

- El apoyo sociotecnológico:

- Contar con un equipo formado por personas provenientes de las áreas social y tecnológica, que apoya a la persona ante emociones que pueden generar los fallos en la tecnología, que resuelve las incidencias en tiempo real o en un breve espacio de tiempo, y que emplea en la comunicación con las personas participantes un lenguaje alejado de tecnicismos, que acerca la tecnología y hace cercana la experiencia.

- Contar con la posibilidad de finalizar la sesión a través de contacto telefónico cuando no es posible hacerlo mediante videoconferencia, de forma que tanto las personas participantes como las y los profesionales de intervención no se queden con la sensación de no poder cerrar de manera adecuada la sesión y despedirse.

- Mostrar una actitud positiva y de seguridad ante las incidencias por parte de las y los profesionales de intervención, tratando de minimizar sus consecuencias, una actitud que contagia a las personas participantes y genera confianza. 


\section{Bibliografía referenciada}

CONDE MORALA, M. (2006): Los cuidados formales a un enfermo de Alzheimer: el gerocultor, Madrid, Asociación para Familias con Alzheimer.

CRESPO, M.; y LÓPEZ, J. (2007): El estrés en cuidadores de mayores dependientes: cuidarse para cuidar, Madrid, Pirámide.

EUSTAT (2002): Escenarios demográficos 2050, VitoriaGasteiz, Eustat [<http://www.eustat.es/elem/ eleo000800/tblooo0866_c.html>].

GOBIERNO VASCO (2011a): Cien propuestas para avanzar en el bienestar y buen trato a las personas que envejecen: bases para un plan de acción, Vitoria-Gasteiz, Eusko Jaurlaritza-Gobierno Vasco.

- (2011b): Estudio sobre las condiciones de vida de las personas mayores de 60 años en la Comunidad Autónoma del País Vasco, Vitoria-Gasteiz, Eusko Jaurlaritza-Gobierno Vasco.
- (2010): Estrategia para Afrontar el Reto de la Cronicidad en Euskadi, Bilbao, Eusko Jaurlaritza-Gobierno Vasco.

- (2009): Plan Estratégico de Servicios Sociales de la CAPV 2011-2014: análisis de la situación de partida, diagnóstico preliminar y propuesta de líneas estratégicas básicas, Vitoria-Gasteiz, Eusko Jaurlaritza-Gobierno Vasco.

- (2007): Los cuidados informales en la Comunidad Autónoma de Euskadi: las necesidades de las personas cuidadoras, Vitoria-Gasteiz, Eusko Jaurlaritza-Gobierno Vasco.

IMSERSO (2005): Libro blanco de la dependencia, Madrid, Imserso.

INE (2013): Cifras de población a 1 de enero de 2013. Resultados definitivos, Madrid, Instituto Nacional de Estadística [hhttp://www.ine.es/ jaxi $/$ menu.do?type $=$ pcaxis \&path $=/$ t20 $/$ p321 $/$ serie $/$ def\&file=pcaxis $)$. 
